\title{
A correlation of pregnancy term, disease activity, serum female hormones, and cytokines in uveitis
}

\author{
C-C Chan, G F Reed, Y Kim, E Agrón, R R Buggage
}

Br J Ophthalmol 2004;88:1506-1509. doi: 10.1136/bjo.2004.048595

\begin{abstract}
Background/aims: Pregnancy and the postpartum period are associated with the activity of autoimmune diseases including uveitis. Although the exact mechanism is unknown, hormones are reported to alter inflammatory cytokines and influence disease activity. The authors studied ocular inflammation, female hormones, and serum cytokine levels during and after pregnancy.

Methods: A prospective, observational case study was conducted. Four pregnant women in their first trimester with chronic non-infectious uveitis were followed monthly until 6 months after delivery. Serum female hormones (oestrogen, progesterone, prolactin) and various cytokines (IL-2, IL-4, IL5, IL-6, IL-10, IFN- $\gamma$, and TGF- $\beta$ ) were measured by ELISA. Results: The four patients had five full term pregnancies. Uveitis activity decreased after the first trimester but flared in the early postpartum period. Serum female hormones, highly elevated during pregnancy, drastically dropped post partum. Cytokine levels except TGF- $\beta$ were mostly undetectable. Conclusion: Female hormones and TGF- $\beta$ may contribute to the activity of uveitis during pregnancy and the postpartum period.
\end{abstract}

$M$ any autoimmune diseases in females are known to improve during pregnancy but worsen in the postpartum period, because pregnancy induces in immune deviation promoting anti-inflammatory cytokines that prevent immunological rejection of the allogenic fetus. ${ }^{12}$ This immunological balance imposed by pregnancy seems to be an antithesis of the immunological imbalance that promotes cell mediated autoimmunity. ${ }^{3}$ Recent evidence indicates that there is a Thl, Th2, and transforming growth factor beta (TGF- $\beta$ ) shift during normal pregnancy and in the postpartum period resulting in a change in autoimmune disease activity. ${ }^{4-6}$ The relation between female hormones, cytokines, and disease activity during and after pregnancy has not been reported in human uveitis. The aim of this study was to assess serum female hormones, cytokines, and ocular inflammation during and after pregnancy.

\section{PATIENTS AND METHODS}

Four women (five full term pregnancies) in their first trimester of pregnancy with non-infectious uveitis for more than 2 years were enrolled in this National Eye Institute institutional review board approved clinical study after providing written informed consent. Patients had monthly ophthalmic examinations until 6 months post partum. At each assessment serum levels of the female hormones (oestrogen, progesterone, and prolactin) and Thl (IL-2 and IFN $\gamma$ ); Th2 (IL-4, IL-5, IL-6, and IL-10); and Th3 (TGF- $\beta$ ) cytokine levels were measured with ELISA. The anti-human antibodies used in the ELISA assay were from the Quantikine kit ( $\mathrm{R} \&$ D Systems, Inc, Minneapolis, MN, USA). TGF- $\beta$ assay measured only the active form.

For examination and presentation of the data, hormone and TGF- $\beta$ values were $\log _{10}$ transformed and averaged by month of gestation and after delivery over the total pregnancies. To quantify the severity of uveitis each eye received one point for the presence of each of the following conditions: keratic precipitates, anterior chamber cells, vitreous cells, vitreous haze, active uveal lesions, active retinal lesions, cystoid macular oedema (CMO), active retinal vasculitis, other inflammatory findings (for example, synechiae), disease flare, or treatment since last study visit. The totals for the patient's eyes were then averaged to obtain the patient's uveitis severity score, which could range from 0 (no conditions present) to a maximum of 11 (all conditions).

To compare mean levels among the early phase of pregnancy (2-4 months), the late phase (5-9 months), and the postpartum phase (delivery to 6 months), a Monte Carlo of estimation of the $p$ value from the permutation test of the hypothesis of no difference between the phases was conducted. Under this hypothesis the monthly sequence of values for each pregnancy is random and as likely to occur as any other. Within each of the five pregnancies the monthly values were randomly permuted. After permutation, mean values (hormone and TGF- $\beta$ levels were $\log _{10}$ transformed before analysis) were calculated by phase for each pregnancy, and these means were then averaged over the five pregnancies by phase. This process was repeated for 100000 iterations, and the fraction of times that the permutation induced difference exceeded the observed difference in absolute value was taken as the point estimate of the two sided $\mathrm{p}$ value. For $95 \%$ confidence against underestimating the $\mathrm{p}$ value, the upper $95 \%$ confidence limit was computed and reported as the $\mathrm{p}$ value. The testing level was two sided and set at 0.05. Since for each outcome variable there were three comparisons made, the Bonferroni criterion-that is, $\alpha=0.05 / 3$, was applied to maintain the control of type I error for multiple comparisons.

Trends in the scatter plots were traced by non-parametric local regression (loess) with the LOESS procedure of the Statistical Analysis System (SAS Institute, Inc, Cary, NC, USA). A local second degree least squares fitting at every point were made with the smoothing parameter (that is, fraction of data in the neighbourhood of a point) automatically selected according the Akaike information criterion.

\section{RESULTS}

The four patients were diagnosed with panuveitis secondary to sarcoidosis, idiopathic granulomatous panuveitis with retinal vasculitis, idiopathic anterior uveitis, and idiopathic posterior uveitis (table 1). The patient with sarcoidosis

Abbreviations: $C M O$, cystoid macular oedema; EAU, experimental autoimmune uveitis; EIU, experimental induced uveitis; TGF- $\beta$, transforming growth factor beta 
Table 1 Clinical data of five pregnancies among four women

\begin{tabular}{|c|c|c|c|c|}
\hline Case & Age & Diagnosis & $\begin{array}{l}\text { Last flare before } \\
\text { pregnancy }\end{array}$ & $\begin{array}{l}\text { Change of medication in early } \\
\text { pregnancy, late pregnancy, post partum }\end{array}$ \\
\hline 1 & $\begin{array}{l}40 \\
41\end{array}$ & Sarcoidosis & $\begin{array}{l}4 \text { years } \\
5 \text { years }\end{array}$ & $\begin{array}{l}\text { decrease, no change, no change } \\
\text { ( }{ }^{*} \text { Score: } 7.45,5.7,4.3,5.3 \text { ) } \\
\text { no change, decrease, increase } \\
\text { (Score: } 5.3,5.3,4.3,6.4 \text { ) }\end{array}$ \\
\hline 2 & 41 & Panuveitis & 2 months & $\begin{array}{l}\text { increase, decrease, increase } \\
\text { (Score: NA, 10.5, 8.9, 11.0) }\end{array}$ \\
\hline 3 & 20 & Iridocyclitis & 5 month & $\begin{array}{l}\text { increase, decrease, increase } \\
\text { (Score: } 7.0,7.5,5.6,3.8 \text { ) }\end{array}$ \\
\hline 4 & 33 & Intermediate uveitis & $>2$ years & $\begin{array}{l}\text { no change, no change, no change } \\
\text { (Score: NA, 5.3,4.3, 6.4) }\end{array}$ \\
\hline \multicolumn{5}{|c|}{$\begin{array}{l}\text { *Uveitis score (before pregnancy, early pregnancy ( } \leqslant 5 \text { months), late pregnancy ( } 6,9 \text { months), postpartum } \\
\text { period). } \\
1 \text { Patients were recruited under an institutional review board approved clinical study after providing written } \\
\text { informed consent. } \\
2 \text { No patients experienced ocular, systemic, or teratogenic complications during the five pregnancies. }\end{array}$} \\
\hline
\end{tabular}

Table 2 Mean of uveitis scores and geometric means of hormone levels

\begin{tabular}{llcrrr}
\hline Phase & Uveitis score & Oestrogen* & Progesterone* & Prolactin* $^{*}$ \\
\hline Early (2-5 months) & 7.4 & 1469 & 15 & 31 & 44838 \\
Mid (5-9 months) & 5.1 & 10911 & 79 & 32225 \\
Post partum (to 6 months) & 6.2 & 244 & 1 & 28 & 38134 \\
\hline \multirow{2}{*}{ *The unit for the hormone and TGF- $\beta$ is $\mathrm{pg} / \mathrm{ml}}$. & & &
\end{tabular}

enrolled for two consecutive pregnancies. No patient developed any systemic medical or obstetric complications during pregnancy or in the postpartum period.

An average of nine (range 7-12) clinical visits were recorded for each pregnancy. Overall, uveitis activity was slightly worse in the first trimester but became either inactive or milder in the late pregnancy, which allowed for reduction of immunosuppressive medications in all patients. However, a slight flare of the uveitis was likely in the first 3 postpartum months.
Cases 1 and 4 had milder uveitis activity (uveitis scores in 0-7 range) while cases 2 and 3 had more severe uveitis (uveitis scores in 3-11 range). The ocular disease in patients with milder uveitis was characterised by trace to $2+$ anterior chamber cells, trace to $1+$ vitreal haze, CMO, inactive chroioretinal lesions, and vasculitis. In contrast, the ocular findings in the two patients with more severe uveitis included 3-4+ anterior chamber cells, 3-4+ vitreal haze, CMO, active chorioretinal inflammatory lesions, and retinal vasculitis.
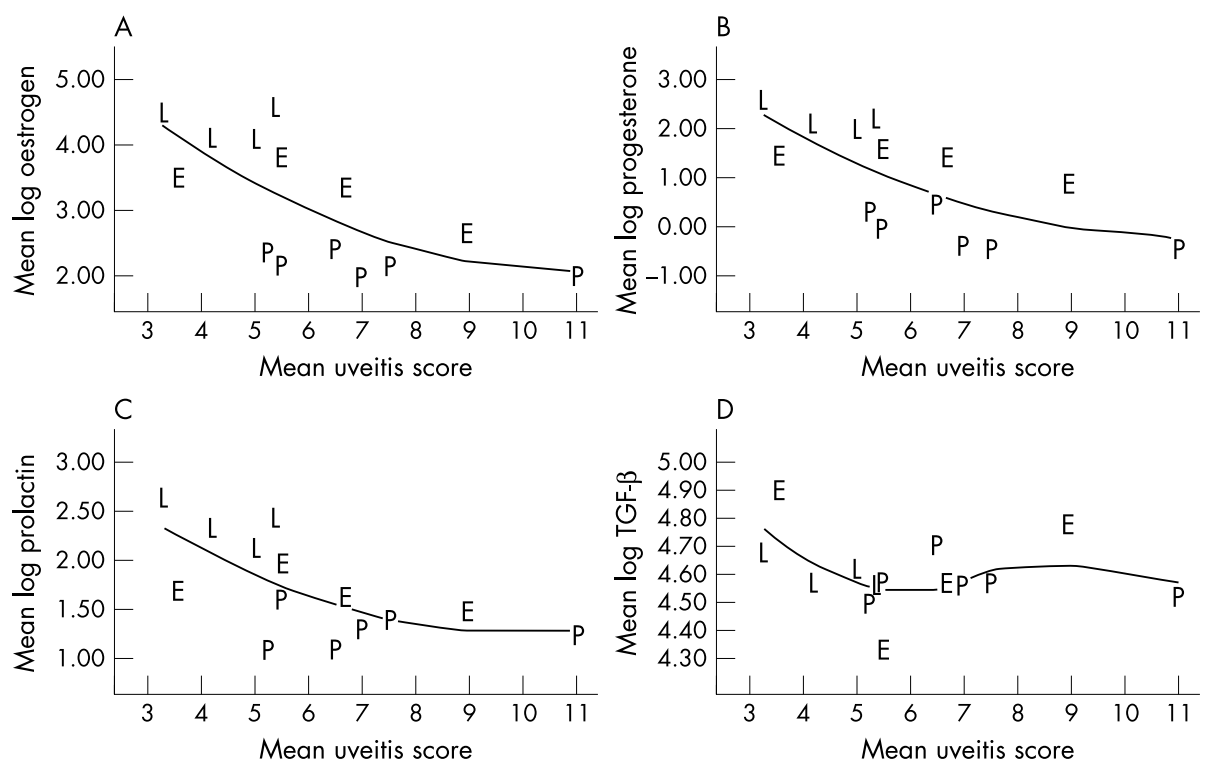

Figures 1 (A-D) Plots of monthly mean $\log _{10}$ of hormones and of TGF- $\beta$ by monthly mean uveitis score. To quantify the severity of uveitis each eye received a point for the presence of each of the following conditions: keratic precipitates, anterior chamber cells, vitreous cell, vitreous haze, active uveal lesions, active retinal lesions, cystoid macular oedema, active retinal vasculitis, other inflammatory findings (for example, synechiae), disease flare, or treatment since last study visit. The totals for the patient's eyes were then averaged to obtain the patient's uveitis severity score, which could range from 0 (no conditions present) to a maximum of 11 (all conditions). The lines are loess fittings that non-parametrically track the relations between the variables. "E" designates early pregnancy, up to month 5 of gestation; " $L$," late gestation, from month 6 to delivery; and "P," postpartum period. 
Table 3 Estimated $p$ values for comparisons between pregnancy phases

\begin{tabular}{|c|c|c|c|c|c|}
\hline Comparison & Uveitis score & Oestrogen & Progesterone & Prolactin & TGF- $\beta$ \\
\hline Early $v$ late & $0.005^{*}$ & 0.025 & 0.126 & 0.002 & 0.069 \\
\hline Early $v$ postpartum & 0.093 & 0.026 & 0.016 & 0.818 & 0.322 \\
\hline Late $v$ postpartum & 0.111 & $<0.001^{*}$ & $<0.001^{*}$ & $<0.001^{*}$ & 0.302 \\
\hline
\end{tabular}

All four patients were on oral prednisone throughout their pregnancies. Two patients (cases 2 and 3 ) required an increased dose of prednisone in early pregnancy, one from $12.5 \mathrm{mg} /$ day to $30 \mathrm{mg} /$ day and the other from $30 \mathrm{mg} /$ day to $40 \mathrm{mg} /$ day. However, later in the pregnancies of these two patients their prednisone dose was decreased to $7.5 \mathrm{mg} /$ day and $17.5 \mathrm{mg} /$ day, respectively. During the late stage of pregnancy, the uvetis scores in these patients dropped into the milder range of 3-6. The two patients with mild uvetis were maintained on low dose prednisone throughout the pregnancy (case 1, $3 \mathrm{mg} /$ day; case 4, $5 \mathrm{mg} /$ day) (table 1 ). In the postpartum period following four of the five pregnancies, there was evidence of disease flare requiring increased immunosuppressive therapy.

As expected, all three female hormones-oestrogen, progesterone, and prolactin-steadily and markedly elevated during pregnancy and drastically dropped in the postpartum period (table 2). Interestingly, most Th1 and Th2 cytokines were below detectable levels. Among a total of 270 assays from all five pregnancies, Th1 and Th2 cytokines were detected in only four assays from three patients with posterior uveitis; the Th2 cytokines during pregnancy (IL-5, $42 \mathrm{pg} / \mathrm{ml}$; IL-6, $291 \mathrm{pg} / \mathrm{ml}$; and IL-10, $40.7 \mathrm{pg} / \mathrm{ml}$; respectively), and the Thl cytokine (IL-2, $42 \mathrm{pg} / \mathrm{ml}$ ) in the first month postpartum. TGF- $\beta$, a Th3 cytokine, was consistently detected in all patients throughout the study.

Table 3 provides estimated $\mathrm{p}$ values for the comparisons of these means between phases, generated by the Monte Carlo permutation test. At the 0.0166 level of testing, uveitis scores dropped significantly from the early to late phase. All hormones decreased significantly from late phase to post partum. Progesterone in the postpartum phase was significantly lower compared to both early and late phases. Prolactin significantly increased from early to late while it significantly decreased from late to post partum. There were no significant differences between phases for TGF- $\beta$.

Although uveitis scores did not vary freely, as they may have been controlled by immunosuppressive therapy, there was a discernible decrease in the female hormones with increasing uveitis score, but no change in TGF- $\beta$ (fig l).

\section{DISCUSSION}

Pregnancy is associated with remediation of many autoimmune diseases including rheumatoid arthritis and multiple sclerosis, yet with exacerbation of other autoimmune conditions such as systemic lupus erythematosis. ${ }^{7-11}$ Few observational publications on non-infectious ocular inflammation have shown improvement during pregnancy and exacerbation after parturition. ${ }^{12-17}$ In a recent large series of 76 pregnancies among 50 women with Vogt-Koyanagi-Harada syndrome, Behçet's disease, and idiopathic uveitis, Rabiah and Vitale observed a flare up within the first 4 months of pregnancy, a relative inactivity in late pregnancy, and a rebound in activity within 6 months of delivery. ${ }^{12}$ We have reported a significantly lower incidence and severity of experimental autoimmune uveitis (EAU) due to higher elevation of circulating TGF- $\beta$ and selective inhibition of Thl responses in pregnant mice, ${ }^{18}$ as well as the effect of sex hormones on cytokine balance in EAU. ${ }^{19}$
This study agrees with other observations demonstrating amelioration of human non-infectious ocular inflammation in mid and late pregnancies and flare up during the postpartum period. Furthermore, we showed the relations among female hormones, TGF- $\beta$, a Th3 cytokine, and disease activity in a small heterogeneous group. These results are compatible with previous reports in EAU and experimental induced uveitis (EIU) models. ${ }^{18-20}$ Although other serum cytokine levels were mostly below the detectable levels, the four measurable Th2 cytokines were found in the pregnancy and the one measurable Thl cytokine was in the postpartum period. The data suggest that physiological changes of sex hormones before and after delivery may polarise the immune response towards a Th2/Th3 response, ${ }^{6}$ which may counterbalance the augmented Thl response observed in noninfectious uveitis.

Data concerning the fluctuation in uveitis status is certainly quite complex. However, one of the main factors is associated with cytokine changes during pregnancy. In general, Th2 cytokines are associated with the downregulation of Thl cytokines and may confer protection from Thlmediated autoimmune diseases. During pregnancy, there is a shift from Th1 to Th2 that occurs both locally, ${ }^{21}$ at the fetal maternal interface, and systemically. ${ }^{22}$ This immune shift is thought to be necessary to avoid fetal rejection, since failure to achieve a Th1 to Th2 immune deviation has been associated with increased risk of spontaneous abortion. ${ }^{5}$ This naturally occurring, systemic shift in immune responses may underlie the improvement in Thl mediated autoimmune diseases including most autoimmune meditated uveitis during pregnancy.

Female patients with uveitis may require close follow up and treatment in the early postpartum period. Pregnancy and pregnancy related hormones including female hormones and cortisol influence the signs and symptoms of autoimmune diseases. $^{423}$ The current study also suggests a correlation between uveitis severity and female hormone levels in the graphic display of the data, although the sample size was too small to achieve statistical significance. Recently oral oestriol therapy has been reported to benefit patients with relapsing remitting multiple sclerosis. ${ }^{24}$ The influence of female hormones on inflammatory cytokines and the interplay between circulating and cellular mechanisms causing uveitis remain to be further investigated.

\section{ACKNOWLEDGEMENTS}

Dr Grace Levy-Clark, Dr Ali Djalilian, and Ms Babilonia Ayukawa assisted with patient care.

\section{Authors' affiliations}

C-C Chan, R R Buggage, Laboratory of Immunology, National Eye Institute, National Institutes of Health, Bethesda, MD, USA

G F Reed, Y Kim, E Agrón, Division of Epidemiology and Clinical Research, National Eye Institute, National Institutes of Health, Bethesda, $M D$, USA

Correspondence to: Chi-Chao Chan, MD, Bldg 10, Room 10N103, NIH/NEI, 10 Center Drive, Bethesda, MD 20892-1857, USA; chanc@ nei.nih.gov

Accepted for publication 17 May 2004 


\section{REFERENCES}

1 Raghupathy R. Th1-type immunity is incompatible with successful pregnancy Immunol Today 1997; 18:478-82.

2 Wilder RL. Hormones, pregnancy, and autoimmune diseases. Ann NY Acad Sci 1998;840:45-50.

3 Makhseed M, Raghupathy R, El-Shazly S, et al. Pro-inflammatory maternal cytokine profile in preterm delivery. Am J Reprod Immunol 2003;49:308-18.

4 Elenkov IJ, Chrousos GP. Stress hormones, proinflammatory and antiinflammatory cytokines, and autoimmunity. Ann N Y Acad Sci 2002:966:290-303

5 Marzi M, Vigano A, Trabattoni D, et al. Characterization of type 1 and type 2 cytokine production profile in physiologic and pathologic human pregnancy Clin Exp Immunol 1996;106:127-33.

6 Olivieri A, De Angelis S, Vaccari V, et al. Postpartum thyroiditis is associated with fluctuations in transforming growth factor-betal serum levels. J Clin Endocrinol Metab 2003;88:1280-4.

7 Khamashta MA, Ruiz-Irastorza G, Hughes GR. Systemic lupus erythematosus flares during pregnancy. Rheum Dis Clin North Am 1997;23:15-30.

8 Ostensen M. Sex hormones and pregnancy in rheumatoid arthritis and systemic lupus erythematosus. Ann N Y Acad Sci 1999;876:131-43, discussion 144

9 Barrett JH, Brennan P, Fiddler $M$, et al. Does rheumatoid arthritis remit during pregnancy and relapse postpartum? Results from a nationwide study in the United Kingdom performed prospectively from late pregnancy. Arthritis Rheum 1999;42:1219-27.

10 Sicotte NL, Liva SM, Klutch R, et al. Treatment of multiple sclerosis with the pregnancy hormone estriol. Ann Neurol 2002;52:421-8.

11 Whitaker JN. Effects of pregnancy and delivery on disease activity in multiple sclerosis. N Engl J Med 1998;339:339-40.

12 Rabiah PK, Vitale AT. Noninfectious uveitis and pregnancy. Am J Ophthalmol 2003; 136:91-8.

13 Hyman BN. Postpartum uveitis. Ann Ophthalmol 1976;8:677-80.
14 Steahly LP. Vogt-Koyanagi-Harada syndrome and pregnancy. Ann Ophthalmol 1990;22:59-62.

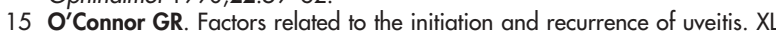
Edward Jackson memorial lecture. Am J Ophthalmol 1983;96:577-99.

16 Nohara M, Norose K, Segawa K. Vogt-Koyanagi-Harada disease during pregnancy. Br J Ophthalmol 1995;79:94-5

17 Chavis PS, Tabbara KF. Effects of pregnancy on the course of uveitis. In: Dodds EM, Couto CA, eds. Uveitis in the third millennium: Elsevier Science 2000:167-70.

18 Agarwal RK, Chan CC, Wiggert B, et al. Pregnancy ameliorates induction and expression of experimental autoimmune uveitis. J Immunol 1999; 162:2648-54.

19 Buggage RR, Matteson DM, Shen DF, et al. Effect of sex hormones on experimental autoimmune uveoretinitis (EAU). Immunol Invest 2003;32:259-73.

20 Miyamoto N, Mandai M, Suzuma I, et al. Estrogen protects against cellular infiltration by reducing the expressions of E-selectin and IL- 6 in endotoxininduced uveitis. J Immunol 1999;163:374-9.

21 Wegmann TG, Lin H, Guilbert L, et al. Bidirectional cytokine interactions in the maternal-fetal relationship: is successful pregnancy a $\mathrm{TH} 2$ phenomenon? Immunol Today 1993;14:353-6.

22 Elenkov IJ, Wilder RL, Bakalov VK, et al. IL-12, TNF-alpha, and hormonal changes during late pregnancy and early postpartum: implications for autoimmune disease activity during these times. J Clin Endocrinol Metab 2001;86:4933-8.

23 Langer-Gould A, Garren H, Slansky A, et al. Late pregnancy suppresses relapses in experimental autoimmune encephalomyelitis: evidence for a suppressive pregnancy-related serum factor. J Immunol 2002;169:1084-91.

24 Soldan SS, Retuerto Al, Sicotte NL, et al. Immune modulation in multiple sclerosis patients treated with the pregnancy hormone estriol. J Immunol 171:6267-74. 